\title{
AUTOPERCEPÇÃO EM SAÚDE BUCAL DE PACIENTES COM HIV/AIDS ACOLHIDOS POR CASA DE APOIO
}

SELF-PERCEPTION IN ORAL HEALTH AMONG HIV/AIDS PATIENTS HOSTED BY A SUPPORT HOUSE

AUTOPERCEPCIÓN EN SALUD BUCAL DE PACIENTES CON VIH/SIDA ACOGIDOS POR UNA CASA DE APOYO

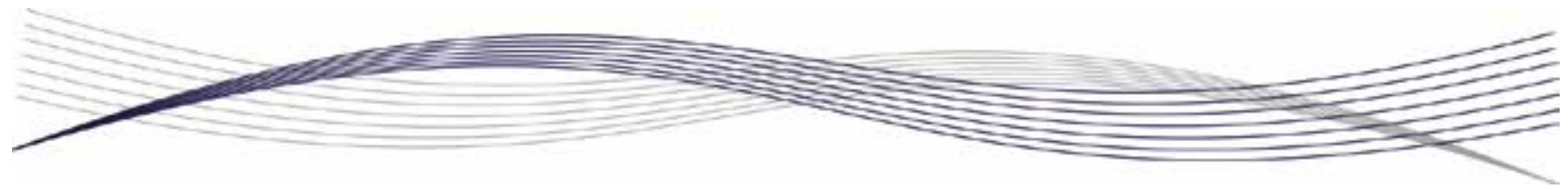

Palavras-chave: Saúde Bucal; HIV; AIDS; Autoimagem.

Keywords: Oral Health; HIV; AIDS; SelfConcept.

Palabras clave: Salud Bucal; VIH; SIDA; Autoimagen.

Submetido: $10 / 11 / 2018$

Aprovado: 06/12/2018

Autor(a) para Correspondência: Jacques Ant. Cavalcante Maciel

R. Lourenço Guimarães, 250 Paulo Ximenes do Prado Groaíras-CE

CEP: 62190-000

E-mail: jacques.maciel@sobral.ufc.br

\section{RESUMO}

Este artigo analisa a autopercepção em saúde bucal de pacientes com vírus da imunodeficiência humana e sindrome da imunodeficiência adquirida (HIV/AIDS) acolhidos pela Casa de Apoio "Madre Anna Rosa Gattorno" em Sobral-CE, descrevendo os fatores relacionados e associando o perfil socioeconômico e educacional ao cuidado em saúde bucal. A coleta de dados foi realizada com aplicação de questionários a 72 pacientes. Quanto às características sociodemográficas, a maioria dos sujeitos do estudo relatou baixas escolaridade e renda. Observou-se que $88,9 \%$ se preocupavam com a saúde bucal; 90,3\% percebiam a influência da condição bucal na saúde geral; e a cárie foi a principal causa de dor. Os resultados sugerem a necessidade de planejar, elaborar e executar serviços odontológicos voltados à promoção da saúde de pacientes com HIV/AIDS, possibilitando a tomada de ações específicas para melhorar a qualidade de vida dessa clientela.

\footnotetext{
1. Cirurgiã-Dentista pela Universidade Federal do Ceará (UFC). Sobral (CE), Brasil. E-mail: amanda_sales_ almeida@hotmail.com

2. Cirurgião-Dentista. Professor no Curso de Odontologia da UFC. Sobral (CE), Brasil. E-mail: jacques.maciel@ sobral.ufc.br

3. Cirurgião-Dentista. Professor no Curso de Odontologia e no Programa de Pós-Graduação em Ciências da Saúde da UFC. Sobral (CE), Brasil. E-mail: fcbbarbosa@yahoo.com.br
} 


\section{ABSTRACT}

This article analyzes self-perception in oral health among patients with human immunodeficiency virus and acquired immunodeficiency syndrome (HIV/AIDS) hosted by the Support House 'Madre Anna Rosa Gattorno' in Sobral, Ceará, Brazil, describing the related factors and associating the socioeconomic and educational profile to oral health care. Data collection was conducted by applying questionnaires to 72 patients. As for the sociodemographic characteristics, most study subjects reported low schooling and income. It was observed that $88.9 \%$ were concerned with oral health; $90.3 \%$ perceived the influence of an oral condition on general health; and caries was the main cause of pain. The results suggest the need to plan, design, and execute dental services aimed at promoting the health of HIV/AIDS patients, making it possible to take specific actions to improve the quality of life of this clientele.

\section{RESUMEN}

Este artículo analiza la autopercepción en salud oral de pacientes con virus de inmunodeficiencia humana y sindrome de inmunodeficiencia adquirida (VIH/SIDA) acogidos por la Casa de Apoyo "Madre Anna Rosa Gattorno" en Sobral, Ceará, Brasil, describiendo los factores relacionados y asociando el perfil socioeconómico y educativo al cuidado en salud bucal. La recogida de datos se realizó mediante aplicación de cuestionarios a 72 pacientes. En cuanto a las características sociodemográficas, la mayoría de los sujetos del estudio informaron bajos niveles de escolaridad e ingresos. Se observó que 88,9\% estaban preocupados por la salud bucal; $90,3 \%$ percibieron la influencia de una condición oral en la salud general; y la caries fue la principal causa de dolor. Los resultados sugieren la necesidad de planificar, diseñar y ejecutar servicios dentales destinados a promover la salud de los pacientes con VIH/SIDA, lo que permite tomar medidas específicas para mejorar la calidad de vida de esta clientela.

\section{INTRODUÇÃO}

Para ser considerado saudável, o indivíduo precisa viver com qualidade de vida ${ }^{1}$. Esse conceito está intimamente relacionado ao de autopercepção, que, em saúde, pode ser entendida como a interpretação das experiências e do estado salubre no contexto da vida diária². A autopercepção em saúde bucal enfoca a importância de como a pessoa percebe sua condição bucal, uma vez que o comportamento é condicionado por essa percepção, pela significância atribuída a ela, por valores culturais e experiências no sistema de saúde. Grande parcela da população não frequenta os serviços odontológicos porque não tem a percepção de sua necessidade e seus benefícios ${ }^{3}$.

Com a mudança do paradigma biomédico, percebeu-se que definições mais atuais de saúde rejeitam a noção de que ela se limita à ausência de doenças físicas, pois é preciso considerar o contexto físico e psicológico, bem como o bem-estar social do indivíduo. No entanto, a saúde bucal continua sendo mensurada por meio de um modelo baseado em agravos ou doenças, com indicadores objetivos e quantitativos, não levando em consideração diversas dimensões envolvidas nem as repercussões dos problemas de saúde na vida diária dos indivíduos ${ }^{4}$.
Dentre as principais doenças crônicas da atualidade se destaca a infecção pelo vírus da imunodeficiência humana (HIV), uma vez que os pacientes tiveram sua sobrevida aumentada com a terapia antirretroviral altamente eficaz (highly active antiretroviral therapy - HAART), o que proporciona significativa melhora da qualidade de vida desses indivíduos ${ }^{5}$. Hoje, a síndrome da imunodeficiência adquirida (AIDS) é considerada uma pandemia. Essa doença deixou de afetar um grupo específico (homossexuais, hemofílicos e usuários de drogas) e passou a ocorrer cada vez mais entre mulheres e crianças ${ }^{1}$.

Segundo dados do Ministério da Saúde ${ }^{6}$, do início de 2007 até junho de 2016 foram notificados 136.945 casos de infecção por HIV no Brasil, sendo 71.396 no Sudeste $(52,1 \%), 28.879$ no Sul $(21,1 \%)$, 18.840 no Nordeste $(13,8 \%), 9.152$ no Centro-0este $(6,7 \%)$ e 6.868 na Região Norte (6,3\%). Em 2015 foram notificados 32.321 casos de infecção por HIV, sendo 2.988 casos no Norte $(9,2 \%), 6.435$ casos no Nordeste $(19,9 \%), 13.059$ no Sudeste $(40,4 \%), 7.265$ no Sul $(22,5 \%)$ e 2.574 no Centro-0este $(8,0 \%)$.

De acordo com a Secretaria de Saúde do Estado do Ceará (Sesa)7, o Ceará ocupa a terceira posição no ranking dos estados do Nordeste, ao lado da Bahia e de Alagoas. De 2007 a 2017, até a Semana 
Epidemiológica (SE) 46 (de 01/01/17 a 18/11/17), foram notificados 6.460 casos de HIV no Ceará. Na série histórica, a taxa de detecção de HIV em adultos desse estado passou de 1,2 casos por $100 \mathrm{mil}$ habitantes em 2007 para 15,8 em 2016. 0 município de Sobral ocupa a primeira posição em notificações no norte do Ceará.

Com o gradativo aumento da população portadora de HIV, mostra-se necessário adotar políticas públicas focadas nos cuidados em saúde dessa clientela, especialmente quando voltadas à prevenção. No contexto odontológico, ainda há escassez de programas e pesquisas envolvendo indivíduos com HIV - que podem tornar-se portadores de severas necessidades em saúde bucal.

0 diagnóstico das condições e a autopercepção da saúde bucal dos indivíduos são cruciais na avaliação dos serviços de saúde e nas estratégias de planejar, elaborar e executar os serviços odontológicos para a promoção da saúde, a prevenção de doenças, - tratamento e a reabilitação dessa população específica.

Para um diagnóstico correto é necessária a compreensão não só de suas necessidades clínicas (objetivas), mas também das relatadas (subjetivas). Para tanto, o paciente com HIV/AIDS precisa ter a oportunidade de expressar seus sentimentos e suas necessidades, que, na maioria das vezes, ficam ocultos e reprimidos no cotidiano das avaliações objetivas da prática clínica e de uma sociedade preconceituosa.

Este artigo analisa a autopercepção em saúde bucal de pacientes com HIV/AIDS acolhidos pela Casa de Apoio “Madre Anna Rosa Gattorno" em Sobral, descrevendo os fatores relacionados e associando o perfil socioeconômico e educacional ao cuidado em saúde bucal.

\section{METODOLOGIA}

Trata-se de estudo exploratório, de caráter descritivo, com abordagem quantitativa, realizado na Casa de Apoio Madre Anna Rosa Gattorno, entidade não governamental pertencente à Rede de Solidariedade Positiva - Núcleo Sobral, consistindo em uma casa de hospedagem temporária que acolhe pacientes com HIV/AIDS do norte do Ceará.

A amostra foi definida por meio de levantamento de todos os pacientes acolhidos pela instituição em análise no período de fevereiro a abril de 2014. 0s critérios de inclusão foram: participação voluntária,

\section{...o paciente com HIV/ AIDS precisa ter a oportunidade de expressar seus sentimentos e suas necessidades...}

confirmada por assinatura do termo de consentimento Livre e esclarecido, e ter 18 anos de idade ou mais. Já os critérios de exclusão foram: severidade da doença que impossibilitasse a entrevista e dificuldade de comunicação que comprometesse o entendimento do questionário.

Os dados foram coletados por meio de questionário adaptado da Pesquisa Nacional de Saúde Bucal (Projeto SB Brasil de 2010). 0 banco de dados construído foi analisado inicialmente no programa computacional Microsoft Excel e posteriormente no programa computacional SPSS, versão 20. As variáveis independentes foram gênero, escolaridade e renda familiar. Para análise de correlação entre as variáveis, utilizou-se o teste de associação do quiquadrado (com nível de significância $p<0,05$ ).

Esta pesquisa teve anuência da Rede de Solidariedade Positiva - Núcleo Sobral e foi aprovada pelo Comitê de Ética em Pesquisa da Universidade Estadual Vale do Acaraú (UVA), sob o Parecer n. 578.448, em conformidade com os princípios da Resolução n. 466/2012, do Conselho Nacional de Saúde $(C N S)^{8}$.

\section{RESULTADOS}

Foram coletados dados de 72 pacientes acolhidos pela casa de apoio. Destes, 34 (47,2\%) eram do gênero masculino e 38 (52,8\%) eram do gênero feminino. Houve predomínio da faixa etária entre 35 e 43 anos de idade, com média de 38,32 anos ( $D P \pm 9,49)$, que correspondeu a $43,1 \%$ da população estudada. Quanto ao estado civil, $50 \%$ dos entrevistados eram solteiros, $36,1 \%$ eram casados e $12,5 \%$ eram viúvos a menor porcentagem correspondeu aos divorciados $(1,4 \%)$.

Houve predomínio de entrevistados com baixa escolaridade: analfabetos $(26,4 \%)$ e indivíduos com Ensino Fundamental incompleto $(48,6 \%)$; apenas $9,7 \%$ tinham Ensino Médio completo. No que concerne ao perfil financeiro, os achados mostraram 
maior participação de indivíduos com renda familiar menor do que 1 salário-mínimo (45,8\%). A maioria dos entrevistados $(68,1 \%)$ não tinha vínculo empregatício, sendo que 37,5\% eram donas de casa, 16,7\% recebiam benefício (auxílio-doença) ou aposentadoria e 13,9\% estavam desempregados (Tabela 1 ).

Tabela 1 - Distribuição dos pacientes por características pessoais. Sobral, 2014.

\begin{tabular}{|c|c|c|}
\hline Caracteristicas & $\mathbf{N}$ & $\%$ \\
\hline \multicolumn{3}{|l|}{ Gênero } \\
\hline Masculino & 34 & 47,2 \\
\hline Feminino & 38 & 52,8 \\
\hline \multicolumn{3}{|l|}{ Faixa etária (anos) } \\
\hline 18 a 24 anos & 7 & 9,7 \\
\hline 25 a 34 anos & 15 & 20,8 \\
\hline 35 a 43 anos & 31 & 43,1 \\
\hline 44 a 53 anos & 15 & 20,8 \\
\hline 54 a 63 anos & 4 & 5,6 \\
\hline \multicolumn{3}{|l|}{ Renda familiar } \\
\hline Menor do que 1 salário-mínimo & 33 & 45,8 \\
\hline 1 salário-mínimo & 23 & 31,9 \\
\hline Maior do que 1 salário-mínimo e menor do que 2 salários-mínimos & 9 & 12,5 \\
\hline 2 salários-mínimos & 3 & 4,2 \\
\hline Maior do que 2 salários-mínimos & 4 & 5,6 \\
\hline \multicolumn{3}{|l|}{ Atividade ocupacional } \\
\hline Desempregado & 10 & 13,9 \\
\hline Dona de casa & 27 & 37,5 \\
\hline Autônomo & 6 & 8,3 \\
\hline Empregada doméstica & 5 & 6,9 \\
\hline Pensionista/aposentado & 12 & 16,7 \\
\hline Agricultor & 7 & 9,7 \\
\hline Auxiliar de serviços gerais (com carteira assinada) & 2 & 2,8 \\
\hline Teólogo & 1 & 1,4 \\
\hline Voluntário de projeto beneficente & 1 & 1,4 \\
\hline Comerciante & 1 & 1,4 \\
\hline \multicolumn{3}{|l|}{ Grau de escolaridade } \\
\hline Analfabeto & 19 & 26,4 \\
\hline Ensino Fundamental incompleto & 35 & 48,6 \\
\hline Ensino Fundamental completo & 6 & 8,3 \\
\hline Ensino Médio incompleto & 4 & 5,6 \\
\hline Ensino Médio completo & 7 & 9,7 \\
\hline Ensino Superior completo & 1 & 1,4 \\
\hline \multicolumn{3}{|l|}{ Estado civil } \\
\hline Solteiro & 36 & 50 \\
\hline Casado/união estável & 26 & 36,1 \\
\hline Divorciado & 1 & 1,4 \\
\hline Viúvo & 9 & 12,5 \\
\hline
\end{tabular}

Fonte: Elaborada pelos autores.

Ao analisar a preocupação com a saúde geral, $93,1 \%$ se mostraram preocupados e 6,9\% estavam despreocupados. Já em relação à visita ao médico para prevenção em saúde, 62,5\% relataram procurar regularmente, principalmente diante da necessidade da realização de consultas e exames de rotina, para melhor acompanhamento do curso clínico da doença. 
Quando questionados sobre a preocupação com a saúde bucal, ou seja, a percepção sobre sua importância, observou-se que $88,9 \%$ se preocupavam. Apenas $29,2 \%$ procuravam regularmente o cirurgiãodentista para prevenção em saúde bucal; os outros só o procuravam quando sentiam dor. Apesar de haver maior preocupação com a saúde geral em comparação com a saúde bucal, a maioria dos pacientes $(90,3 \%)$ percebe a influência da saúde bucal na saúde geral, mostrando uma autopercepção positiva da saúde bucal. Dentre os fatores relacionados a essa influência, os pacientes citaram a halitose e o edentulismo.

Ao avaliar a presença de algum problema na boca e a preocupação do paciente, observou-se que $55,6 \%$ já se sentiram preocupados devido à presença de enfermidades que surgiram após a infecção por HIV. A maioria $(91,7 \%)$ relatou ter sentido dor ou desconforto ao comer algum tipo de alimento. As principais causas dessa dor ou desconforto foram cárie $(65,3 \%)$, afta $(12,5 \%)$, candidíase $(6,9 \%)$ e gengivite $(5,6 \%)$ (Figura 1$)$.

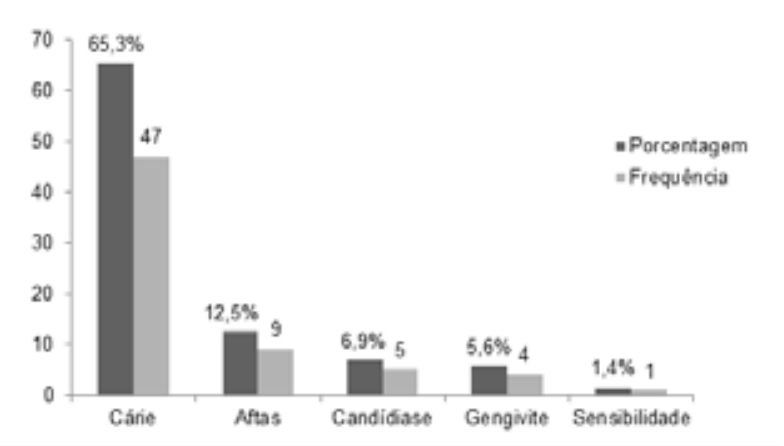

Figura 1. Causas de dor ou desconforto para comer algum tipo de alimento. Sobral, 2014.

Fonte: Elaborada pelos autores.

Quanto à percepção da preocupação com a saúde bucal, as variáveis indicaram correlação estatisticamente significativa apenas para o grau de escolaridade $(p=0,037)$, ou seja, quanto mais alto o nivel educacional, maior a preocupação com a saúde bucal. Já as variáveis sexo $(p=0,182)$ e renda familiar $(p=0,910)$, não apresentaram correlação estatisticamente significativa.

Constatou-se significância estatística $(p=$ $0,032)$ na correlação entre a influência da condição bucal na saúde geral e o gênero, ou seja, o gênero feminino se associou a maior percepção da influência da saúde bucal na saúde geral. No que concerne

\section{A autopercepção em saúde bucal tem sido relacionada ao modelo de determinação social da doença...}

ao grau de escolaridade $(p=0,100)$ e à renda familiar $(p=0,409)$, não foi encontrado resultado estatisticamente significativo.

Em relação ao cuidado com a saúde bucal após a infecção por HIV, observou-se que $55,5 \%$ passaram a se cuidar mais, $41,7 \%$ não mudaram seus hábitos e $2,8 \%$ passaram a se cuidar menos. Ao serem questionados quanto à satisfação com a aparência da boca, $61,1 \%$ se mostraram satisfeitos e $38,9 \%$ relataram insatisfação. Analisando a frequência de escovação dos dentes, $80,6 \%$ dos indivíduos afirmaram que realizam sua higiene oral 2 ou 3 vezes por dia.

A avaliação do cuidado com a saúde bucal após a infecção por HIV não constatou associação estatisticamente significativa com qualquer das variáveis estudadas: gênero $(p=0,994)$, renda familiar $(p=0,173)$ e grau de escolaridade $(p=$ $0,907)$.

E em relação ao acesso a serviços odontológicos, $56,9 \%$ afirmaram ter fácil acesso ao dentista. Sobre a forma desse acesso: 30,6\% apontaram a Atenção Primária à Saúde (APS), 18,1\% indicaram o projeto de extensão universitária "Sorriso Positivo", vinculado à Universidade Federal do Ceará (UFC), e $8,3 \%$ mencionaram serviços privados.

\section{DISCUSSÃO}

A análise dos fatores relacionados à autopercepção em saúde bucal entre pacientes com HIV/AIDS se mostra relevante para a atuação dentro dos determinantes sociais da saúde com vistas à redução de iniquidades em saúde bucal. Neste estudo, o grupo de pacientes acolhido pela Casa de Apoio Madre Ana Rosa Gattorno apresentou autopercepção positiva em saúde bucal.

A autopercepção em saúde bucal tem sido relacionada ao modelo de determinação social da doença, o que envolve fatores econômicos, educacionais e culturais. Políticas de saúde focadas na concepção exclusivamente biomédica da doença 
contribuem para uma assistência odontológica ineficaz, como consequência, a longo prazo a prevalência do edentulismo, comprometendo a qualidade de vida9.

Além da insatisfação com a aparência física, a total ausência de dentes afeta negativamente a alimentação e implica incapacidade física, pois limita a escolha dos alimentos e prejudica a nutrição e a saúde geral ${ }^{10}$. Isso é preocupante para o paciente com HIV/AIDS, uma vez que a alimentação adequada se mostra crucial para a resposta positiva ao tratamento antirretroviral na vida diária ${ }^{11}$.

Um estudo sobre os cuidados odontológicos de pacientes com HIV/AIDS traz a halitose como fator importante da autopercepção negativa em saúde bucal - um aspecto diferente do observado neste estudo. Ainda assim, a abordagem da halitose tem relevância por se tratar de uma condição geradora de problemas sociais e psicológicos: sua ocorrência causa sérios prejuízos na qualidade de vida ${ }^{12}$. 0 mau odor bucal se torna fator significativo nas relações sociais, podendo gerar preocupação não só em termos de saúde física, mas também de saúde mental, com alterações psicológicas que levam ao isolamento social ${ }^{13}$. As pessoas soropositivas enfrentam dificuldades para se sentirem aceitas socialmente, o que aumenta o medo e, sobretudo, o isolamento dessa clientela ${ }^{14}$, o que se torna ainda pior quando associado a halitose.

A saúde bucal está fortemente associada à saúde física e mental e há significativo aumento das necessidades de saúde bucal em pessoas com infecção por HIV ${ }^{15}$. A AIDS leva a progressiva imunossupressão, resultando em maior suscetibilidade do organismo a infecções oportunistas. Quando combinada a más condições bucais, pode prejudicar ainda mais o bemestar do paciente, contribuindo para piorar sua qualidade de vida ${ }^{16}$.

A boca não pode ser tratada isoladamente: a saúde bucal assume tanta importância para garantir um organismo sadio quanto a saúde geral para causar problemas na boca do paciente. A cavidade oral é porta de entrada para micro-organismos causadores de diversas doenças sistêmicas. E é notório que muitas doenças do corpo têm manifestações bucais, constituindo sinal para diagnosticar problemas de saúde geral ${ }^{17}$.

A frequência adequada de escovação dos participantes deste estudo se contrapõe às observações de outros estudos dedicados a pacientes com HIV/AIDS, pois a saúde bucal quase sempre é

\section{As pessoas \\ soropositivas enfrentam \\ dificuldades para \\ se sentir aceitas \\ socialmente...}

subvalorizada por pacientes cujo foco recai sobre o tratamento da doença sistêmica ${ }^{18}$. A cavidade oral, quando não se encontra em condição saudável ou não é higienizada do modo correto, passa a ser um foco de infecção que pode afetar negativamente o curso clínico da doença principal, com potencial surgimento de infecções oportunistas ${ }^{19}$.

As manifestações orais da infecção por HIV são comuns e podem representar os primeiros sinais clínicos da doença, às vezes antecedem as manifestações sistêmicas ${ }^{20}$. A microbiota oral de indivíduos imunocompetentes é diferente da flora de pacientes com HIV/AIDS. A associação de medicamentos, inclusive antibióticos, corticosteroides e anti-inflamatórios, adotada no tratamento pode propiciar a proliferação de várias espécies de fungos, como a Candida, que causa a candidíase oral ${ }^{21}$. Esta é a manifestação bucal mais frequente em portadores de HIV, devido à alteração da quantidade de Linfócitos T, sendo, portanto um indicador da debilitação do sistema imunológico 22 .

Os numerosos efeitos adversos da terapia antirretroviral podem contribuir para uma higiene bucal inadequada e uma dieta inapropriada, aumentando o risco de cárie ${ }^{23}$ - o problema de saúde bucal mais relatado pelos participantes. Ainda que o estudo não tenha recorrido a exame clínico para avaliar a prevalência e severidade da doença, o autorrelato corrobora outros estudos indicativos de que os indivíduos com HIV/AIDS apresentam alta prevalência de cárie e gengivite, devido à influência de fatores crônicos envolvidos na infecção por HIV, como: a) uso prolongado de medicamentos açucarados; b) alterações do fluxo salivar pelo uso de medicamentos e/ou alterações das glândulas salivares; c) dieta rica em carboidratos; d) repetidos episódios de internação; e e) higiene oral deficiente. Isso se assemelha aos dados relatados neste estudo pelos pacientes ${ }^{24}$.

As medidas de autopercepção da saúde bucal envolvem dimensões comuns, como a aparência. 
A preocupação com a aparência se torna um fator relevante nas relações humanas. Um sorriso bonito, com a presença de dentes brancos e alinhados, aumenta a autoestima do indivíduo, já o indivíduo desprovido dessa condição sente-se excluído da sociedade 25 . No nível psicossocial, uma boca sadia garante a manutenção da boa aparência e da comunicação interpessoal, assim, é um fator de relativa importância na preservação da autoestima e autoconfiança, especialmente com o aumento da expectativa de vida dos infectados por HIV ${ }^{26-27}$.

A saúde bucal se mostra fundamental para essa clientela, pois há maior probabilidade de infecções que geram situações dolorosas, dificultando a alimentação e, consequentemente, interferindo na qualidade de vida do indivíduo. Além disso, tais infecções podem ser de difícil tratamento, seja por causa dos micro-organismos mais poderosos ou da baixa imunidade ${ }^{28}$.

Apesar da reconhecida importância da saúde bucal, cerca de $18 \%$ das pessoas nunca foram ao dentista até os 12 anos, constituindo uma parcela significativa da população brasileira sem acesso a serviços odontológicos. 0 uso dos serviços odontológicos do Sistema Único de Saúde (SUS) tem sido um desafio para a odontologia, com consequente comprometimento da integralidade e resolutividade da assistência em termos de saúde bucal ${ }^{29}$. 0 conceito de "acesso aos serviços de saúde" tem amplo escopo, representando o grau de adequação entre as necessidades de saúde, a demanda e o uso de serviços de saúde. Implica garantia de ingresso no sistema de saúde, de modo que os indivíduos possam usufruir dos serviços em caráter contínuo e organizado ${ }^{6}$. Nesse sentido, o acesso aos serviços é complexo e relaciona-se à percepção das necessidades de saúde e da situação clínica atual ${ }^{29}$.

Muitos dos pacientes que afirmaram ser a APS a principal porta de entrada para os serviços odontológicos não revelam seu diagnóstico ao cirurgião-dentista, por receio de ser discriminados. Sabe-se que muitos profissionais se sentem inseguros ao atender essa clientela, devido ao risco pessoal de contágio e às dúvidas quanto à eficácia das medidas de controle da infecção cruzada. A adequada avaliação do risco ocupacional diante do HIV constitui fator importante na disposição de profissionais de saúde para o atendimento de pessoas com HIV/AIDS ${ }^{30}$. Assim, o preconceito e a discriminação contra pessoas soropositivas são as maiores barreiras no combate à epidemia, ao

\section{...iniciativas \\ inerentes à saúde \\ bucal são vitais \\ para os pacientes \\ com HIV/AIDS...}

seu diagnóstico, ao adequado apoio e à atenção e assistência odontológica.

0 Projeto Sorriso Positivo foi citado por alguns pacientes como forma de acesso aos serviços odontológicos. Trata-se de uma atividade de extensão universitária do Curso de Odontologia da UFC em Sobral que presta assistência odontológica aos pacientes com HIV/AIDS na Casa de Apoio de Madre Anna Rosa Gattorno, um espaço de acolhimento e cuidado que respeita o indivíduo em sua integralidade. Desde sua criação, a demanda de atendimentos tem sido relevante, o que ratifica sua contribuição para a melhoria da qualidade de vida. 0 diferencial do projeto é mostrar aos pacientes a importância da saúde bucal no curso clínico da AIDS, promovendo uma autopercepção positiva da saúde bucal. Portanto, iniciativas inerentes à saúde bucal são vitais para os pacientes com HIV/AIDS, como as ações coletivas do projeto, por meio de métodos educativos e preventivos.

A manutenção de uma boa saúde bucal no tratamento desse grupo específico de pacientes é indispensável que haja a integração da promoção de saúde bucal aos programas de saúde pública voltados aos pacientes com retroviroses, com vistas a melhor qualidade de vida e saúde geral dessa clientela, com consequente aumento da expectativa de vida.

\section{CONCLUSÃO}

Conclui-se que a população deste estudo é composta por indivíduos com baixa escolaridade e renda e a maioria apresenta autopercepção positiva da saúde bucal, reconhecendo sua importância no contexto da saúde geral. Os fatores associados a tal autopercepção foram o edentulismo e a halitose, que afetam negativamente o convívio social e a qualidade de vida dos indivíduos.

A maioria dos participantes não procura 0 dentista para prevenção, a busca pelos serviços odontológicos ainda decorre principalmente de dor ou desconforto, o que prejudica as atividades da 
vida diária em virtude de procedimentos clínicos com enfoque curativista e/ou mutilador.

Pode-se afirmar que este estudo reforça a importância de analisar a percepção da saúde bucal pelos pacientes com HIV/AIDS e representa contribuição relevante para o desenvolvimento de programas educativos e preventivos voltados a esse grupo populacional específico.

\section{CONTRIBUIÇÃO DOS AUTORES}

Amanda Sales de Almeida contribuiu com a realização da pesquisa, o delineamento do estudo e a redação do manuscrito. Jacques Antonio Cavalcante Maciel e Francisco César Barroso Barbosa contribuíram com a redação e revisão crítica do manuscrito.

\section{REFERÊNCIAS}

1. Lima ALO, Albuquerque VWT, Silva JIBW, Peixoto FB, Ferreira SMS. Percepção sobre saúde bucal de mulheres vivendo com HIV/AIDS. Revista Semente [serial on the internet]. 2011 [cited 2018 Dec 11];6(6):117-30. Available from: file:// LD:/150-1-548-1-10-20130413.pdf

2. Gerritsen $A E$, Allen PF, Witter DJ, Bronkhorst EM, Creugers NH. Tooth loss and oral health-related quality of life: a systematic review and metaanalysis. Health Qual Life Outcomes [serial on the internet]. 2010 [cited 2018 Dec 11];(8):126. Available from: https://www.researchgate.net/ publication/47678098 Tooth loss and oral health-related quality of life A systematic review and meta-analysis

3. Buczynski AK, Castro GF, Souza IPR. 0 impacto da saúde bucal na qualidade de vida de crianças infectadas pelo HIV: revisão de literatura. Ciênc Saúde Colet [serial on the internet]. 2008 [cited 2018 Dec 11];13(6):1797-805. Available from: http://www.scielo.br/pdf/csc/v13n6/a14v13n6.pdf

4. Velasquez JR. Atención odontológica al paciente con VIH. Odontol Mod. 2012;8(91):14-5.

5. Koyio L, Ranganathan K, Kattappagari KK, Williams DM, Robinson PG. Oral health needs assessment world-wide in relation to HIV. Themes: oral health needs and inequalities, oral health promotion, coordinating research and enhancing dissemination in relation to HIV - a workshop report. Oral Dis. $2016 ; 22$ (Suppl 1):199-205.

6. Brasil. Boletim Epidemiológico HIV/AIDS 2016 [serial on the internet]. 2017 [cited 2018 Dec 11];48(1):1-52. Available from: http:// portalarquivos2.saude.gov.br/images/pdf/2017/ janeiro/05/2016 034-Aids publicacao.pdf

7. Ceará (Estado). Boletim epidemiológico HIV/ AIDS, dezembro 2017. Fortaleza: Secretaria da Saúde; 2017.

8. Brasil. Resolução n. 466/2012, de 12 de dezembro de 2012 [document on the internet]. 2012 [cited 2018 Dec 11]. Available from: https://www.fop. unicamp.br/index.php/pt-br/docentes/fop/459resolucao-cns-466-2012

9. Montero J, Castillo-0yagüe R, Lynch CD, Albaladejo A, Castaño A. Self-perceived changes in oral healthrelated quality of life after receiving different types of conventional prosthetic treatments: a cohort follow-up study. J Dent. 2013;41:493-503.

10. Listl S, Moeller J, Manski R. A multi-country comparison of reasons for dental non-attendance. Eur J Oral Sci. 2014;122(1):62-99.

11. Apelian N, Vergnes JN, Bedos C. Humanizing clinical dentistry through a person-centred model. International Journal of Whole Person Care [serial on the internet]. 2014 [cited 2018 Dec 11];1(2):3050. Available from: http://ijwpc.mcgill.ca/article/ view $/ 2 / 12$

12. Gomes AMT, Silva EMP, Oliveira DC. Representações sociais da AIDS para pessoas que vivem com HIV e suas interfaces cotidianas. Rev Latinoam Enferm [serial on the internet]. 2011 [cited 2018 Dec 11];19(3):485-92. Available from: http://www. scielo.br/pdf/rlae/v19n3/pt 06

13. Leao JC, Ribeiro CMB, Carvalho AAT, Frezzini C, Porter S. Oral complications of HIV disease. Clinics [serial on the internet]. 2009 [cited 2018 Dec 11];64(5):459-70. Available from: https://www. ncbi.nlm.nih.gov/pmc/articles/PMC2694251/

14. Sánchez GA, D'Eramo LR, Lecumberri R, Squassi AF. Impact of oral health care needs on healthrelated quality of life in adult HIV+ patients. Acta Odontol Latinoam. 2011;24(1):92-7.

15. Soares GB, Garbin CA, Rovida TA, Garbin AJ. Oral health associated with quality of life of people living with HIV/AIDS in Brazil. Health Qual Life Outcomes [serial on the internet], 2014 [cited 2018 Dec 11];1(12):28. Available from: https://www.ncbi. $\mathrm{nlm}$.nih.gov/pmc/articles/PMC3942772/

16. Rwenyonyi CM, Kutesa A, Muwazi L, Okullo I, Kasangaki A, Kekitinwa A. Oral manifestations in HIV/AIDS-infected children. Eur J Dent [serial on the internet]. 2011 [cited 2018 Dec 11];5(3):291-8. Available from: https://www.ncbi.nlm.nih.gov/pmc/ articles/PMC3137442/

17. Brasil. Hepatite, AIDS, herpes na prática 
odontológica. Brasília (DF): Ministério da Saúde; 1994.

18. Lambert RF, Orrell C, Haberer JE. "It was pain. That's it. It was pain." Lack of oral health care among otherwise healthy young adults living with HIV in South Africa: a qualitative study. Plos One [serial on the internet]. 2017 [cited 2018 Dec 11];12(12):e0188353. Available from: https:// journals.plos.org/plosone/article?id=10.1371/ journal.pone. 0188353

19. Manikandan S, Suresh Kumar V, Ramesh K. Oral manifestations of HIV infected children undergoing highly active antiretroviral therapy. J Pierre Fauchard Acad. 2013;27:49-52.

20. Souza AJ, Gomes-Filho IS, Silva CAL, PassosSoares JS, Cruz SS, Trindade SC, et al. Factors associated with dental caries, periodontitis and intra-oral lesions in individuals with HIV/AIDS. AIDS Care. $2017 ; 30(5): 578-85$.

21. Massarente DB, Domaneschi C, Marques HH, Andrade SB, Goursand D, Antunes J. Oral health-related quality of life of paediatric patients with AIDS. BMC Oral Health [serial on the internet]. 2011 [cited 2018 Dec 11];5(11):2. Available from: https://bmcoralhealth.biomedcentral.com/ articles/10.1186/1472-6831-11-2

22. Herkrath FJ, Vettore MV, Werneck GL. Contextual and individual factors associated with dental services utilisation by Brazilian adults: a multilevel analysis. Plos 0ne [serial on the internet]. 2018 [cited 2018 Dec 11];13(2):e0192771. Available from: https:// journals.plos.org/plosone/article?id=10.1371/ journal.pone.0192771

23. Loignon C, Landry A, Allison P, Richard L, Bedos C. How do dentists perceive poverty and people on social assistance? A qualitative study conducted in Montreal, Canada. J Dent Educ. 2012;76:545-52.

24. Luxford K, Safran DG, Delbanco T. Promoting patient-centered care: a qualitative study of facilitators and barriers in healthcare organizations with a reputation for improving the patient experience. Int J Qual Health Care. 2011;23:510-5.

25. Gabardo MCL, Moysés S. Autopercepção de saúde bucal conforme o Perfil de Impacto da Saúde Bucal (OHIP) e fatores associados: revisão sistemática. Rev Panam Salud Pública [serial on the internet]. 2013 [cited 2018 Dec 11];33(6):439-45. Available from: https://www.scielosp.org/article/rpsp/2013. v33n6/439-445/

26. Ramphoma KJ, Naidoo S. Knowledge, attitudes and practices of oral health care workers in Lesotho regarding the management of patients with oral manifestations of HIV/AIDS. South African Dental Journal. 2014;69(10):448-53.
27. Viana PAS, Novais CT, Reis RWC, Flor SMC, Rosa PB. Aspectos epidemiológicos, clínicos e evolutivos da AIDS em idosos no Norte do Ceará. Sanare (Sobral, Online) [serial on the internet]. 2017 [cited 2018 Dec 11];16(2):31-6. Available from: https://sanare. emnuvens.com.br/sanare/article/view/1175

28. Al-Sandook T, Al-Naimi R, Younis M. An investigation of dentists' knowledge, attitude and practice towards HIV+ and patients with other blood borne viruses in Mosul city. Al-Rafidain Dental Journal. 2013;10(2):298-308.

29. Brasil. SB Brasil: Projeto Pesquisa Nacional de Saúde Bucal - resultados principais. Brasília (DF): Ministério da Saúde; 2011.

30. Mohamed N, Saddki N, Yusoff A, Jelani AM. Association among oral symptoms, oral healthrelated quality of life, and health-related quality of life in a sample of adults living with HIV/AIDS in Malaysia. BMC Oral Health [serial on the internet]. 2017 [cited 2018 Dec 11];17:119. https://www.ncbi. nlm.nih.gov/pmc/articles/PMC5568203/
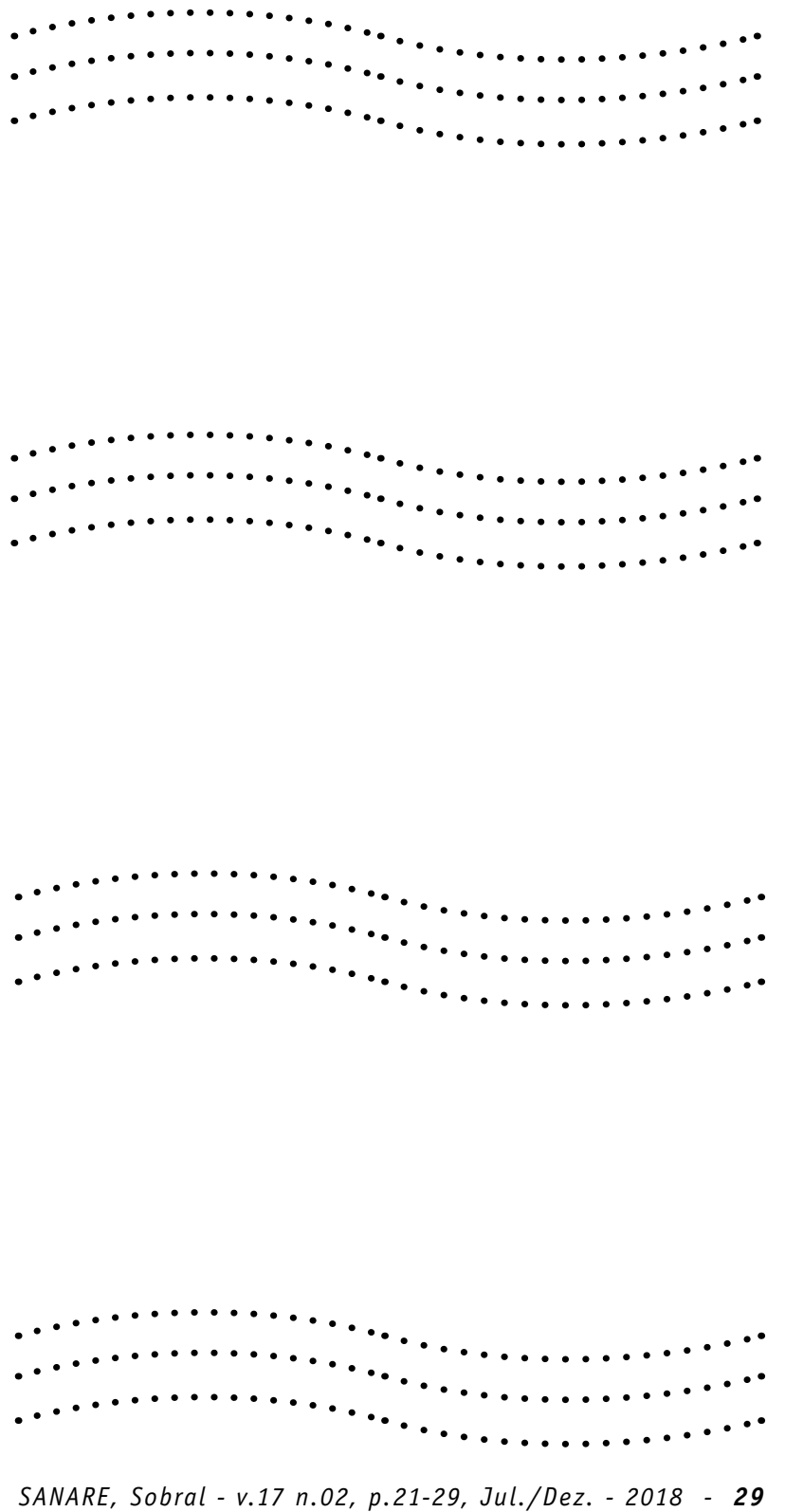